\title{
AMIDOS FOSFATADOS DA SWARTZIA BURCHELLI: PROPRIEDADES FÍSICAS E FÍSICO-QUÍMICAS
}

\author{
ALLINE EMANNUELE CHAVES RIBEIRO' 1 \\ DIEGO PALMIRO RAMIREZ ASCHERI ${ }^{2}$ \\ JOSÉ LUIS RAMÍREZ ASCHERI ${ }^{3}$
}

\begin{abstract}
A Swartzia burchelli é uma Leguminosae que ocorre no bioma do Cerrado brasileiro, conhecida como "banha-de-galinha". O amido contido em suas sementes podem ser aproveitado para diversos fins tecnológicos, porém, requer modificações para seu uso específico. O objetivo deste trabalho foi estudar as características do amido fosfatado da $S$. burchelli em função da concentração de ácido tripolifosfato de sódio (3, 5 e $7 \%$ ) e do tempo de fosfatação (30, 40 e $50 \mathrm{~min}$ ). O experimento foi conduzido em esquema fatorial $2^{2}$ com três pontos centrais, analisado por meio de Metodologia de Superfície de Resposta. Dos amidos foram determinados sua composição química, fósforo e cinzas, forma e tamanho, difração de raios- $X$, espectroscopia na região do infravermelho, viscosidade de pasta e índices de solubilidade em água e de absorção de água. A adequação de um modelo polinomial de segunda ordem foi testada por meio de análise de variância para estabelecer uma equação preditiva dos dados experimentais. As respostas também foram analisadas aplicando correlação de Pearson e análises de componentes principais. Diferentes tamanhos, graus de cristalinidade, gelatinização e empastamento, solubilidades em água e absorção de água foram observados devido à natureza do amido nativo ou pelo processo de fosfatação. O amido fosfatado por 40 min com $5 \%$ de ATPS apresentou menor retrogradação e quebra de viscosidade e maior transmitância; enquanto que o amido fosfatado por 50 min com $7 \%$ de ATPS apresentou maior teor de cinzas e fósforo, grau de cristalinidade, comprimento e largura de seus grânulos e maior temperatura de pasta.
\end{abstract}

PALAVRAS-CHAVE: FRUTOS DO CERRADO; ANALIZADOR RÁPIDO DE VISCOSIDADE; RAIOS-X; PODER DE INCHAMENTO E ÍNDICE DE SOLUBILIDADE; METODOLOGIA DE SUPERFÍCIE DE RESPOSTA.

1 MSc. em Engenharia Agrícola, Curso de Mestrado de Engenharia Agrícola, UEG, Anápolis, GO (e-mail: allineribeiroqi@ gmail.com).

2 Doutor em Engenharia de Alimentos, Curso de Mestrado de Engenharia Agrícola, UEG, Anápolis, GO (email: ascheridpr@gmail.com).

3 Doutor em Ciências de Alimentos, Embrapa Agroindústria de Alimentos, Guaratiba, RJ (e-mail: jose.ascheri@embrapa. br). 


\section{INTRODUÇÃO}

O amido além de se encontrar abundantemente na natureza, ser de baixo custo, renovável e biodegradável, é um biopolímero muito importante na agroindústria. Amidos convencionais de milho e mandioca são utilizados, por exemplo, na produção de biocombustíveis (MOJOVIĆ et al., 2006; LATNER, O'KRAY \& JIANG, 2006; PRIELER \& FISCHER, 2009), plásticos biodegradáveis de coberturas do solo de cultivo (KASIRAJAN \& NGOUAJIO, 2012), filmes e coberturas biodegradáveis para frutas e sementes (BOURTOOM, 2008; DHANAPAL et al. 2012). Na indústria de alimentos, entre outras funções, são utilizados para conferir textura, espessante, fornecedor sólidos em suspensão e proteger os alimentos durante o processamento (ASCHERI et al., 2010).

No entanto, a utilização desses amidos convencionais na produção de gêneros não alimentícios acaba encarecendo-os e gerando competição com caráter alimentar. Por esse motivo, atualmente procura-se novas fontes de amido para o consumo humano.

A Swartzia burchelli, leguminosa do Cerrado brasileiro, popularmente conhecida como "banha-de-galinha", pacová de macaco, jacarandá-banana, jacarandá-de-sangue e banana-depapagaio (MANSANO, LEWIS \& TOZZI, 2002), apresenta frutos com sementes grandes contendo amido. Esse amido e sua modificação química ainda não foram estudados. A produção de amidos modificados é uma alternativa que vem sendo desenvolvida com o objetivo de superar limitações tecnológicas de novos amidos, aumentando, assim, a utilidade deste polímero nas diversas aplicações agroindustriais.

As propriedades físico-químicas e funcionais dos amidos são influenciadas pela fonte botânica que o originou (SINGH et al., 2003, SRICHUWONG et al., 2005, XIAO et al, 2011), e a estruturas granular e molecular (SINGH et al., 2003, LINDEBOOM, CHANG \& TYLER, 2004), por isso, espera-se que as propriedades do amido de $S$. burcelli, sejam bem diferentes dos amidos convencionais. As propriedades mais importantes estudadas nos amidos são a forma e tamanho dos grânulos, transparência e opacidade, gelatinização e retrogradação, solubilidade, inchamento, absorção em água, sinérese e comportamento reológico de suas pastas e géis moleculares (SINGH et al., 2003, SRICHUWONG et al., 2005, HERNÁNDEZ-MEDINA et al., 2008).

Amidos com grânulos pequenos $(2,0 \mu \mathrm{m})$ podem ser usados como substitutos de gordura devido ao tamanho ser semelhante ao dos lipídeos (LEONEL, 2007). As pastas se tornam mais opacas quanto maior o teor de amilose nos grânulos de amido (CEREDA, 2002). A amilopectina contribui para o inchamento do grânulo, enquanto a amilose e os lipídios o inibem. A estrutura cristalina depende do tipo e grau de associação intermolecular existente entre a amilose e a amilopectina (SINGH et al., 2003). Essa estrutura é destruído gradual e irreversivelmente durante o processo de gelatinização (HERNÁNDEZ-MEDINA et al., 2008), por isso, a temperatura de gelatinização é característica para cada tipo de amido e depende fundamentalmente da transição vítrea da fração amorfa.

A viscosidade é uma das propriedades tecnológicas mais importantes dos amidos. A curva de viscosidade representa o seu comportamento durante o aquecimento e permite avaliar as características da pasta formada devido às modificações estruturais das moléculas de amido e a tendência a retrogradação durante o resfriamento. Devido à retrogradação se potencializar em temperaturas de refrigeração, a sinérese é frequente em produtos refrigerados e congelados. É importante considerar que amidos de diferentes fontes botânicas e segundo a sua relação de amilose/amilopectina retrogradam em diferentes valores de temperatura (FRANCO et al., 2001).

A modificação física ou química do amido também promove a alteração das suas propriedades. A acidificação com tripolifosfato de sódio (ATPS), um dos tipos de modificação química mais utilizada em amidos por ser um processo simples e econômico, produz pastas com boa claridade (BATISTA et al., 2010). Autores como Noda et al. (2007); Limberger et al. (2008); Batista et al. (2010), Pereira \& Ascheri (2011) deram especial atenção à modificação de amidos por fosfatação, registrando bons resultados em suas propriedades físicas, físico-químicas e reológicas. 
Nesse contexto, o presente trabalho teve por objetivo modificar quimicamente o amido com ácido tripolifosfato de sódio em diferentes concentrações e tempos reacionais, analisar as características físicas, físico-químicas e químicas dos amidos nativo e modificados, visando estabelecer indicativos de sua qualidade e utilidade tecnológica e fornecer informações sobre o aproveitamento desse amido.

\section{MATERIAL E MÉTODOS}

Os experimentos foram realizados nos laboratórios de Química da Unidade Universitária de Ciências Exatas e Tecnológicas da Universidade Estadual de Goiás (Anápolis-GO), e Tecnologia de Cereais da Empresa Brasileira de Pesquisa Agropecuária - Agroindústria de Alimentos (Rio de Janeiro-RJ), entre os meses de outubro de 2012 e julho de 2013.

Os frutos da S. burchelli foram colhidos de plantas sadias localizadas no Cerrado goiano, no perímetro rural do município de Porangatu (GO), com latitude $13^{\circ} 26^{\prime} 27^{\prime \prime} \mathrm{S}$, longitude $49^{\circ} 08^{\prime} 55^{\prime \prime} \mathrm{W}$ e altitude de $396 \mathrm{~m}$. O critério da seleção dos frutos foi a cor amarelada da casca, inteiros e sadios. Após a seleção os frutos foram embalados em caixas de papelão e transportados ao Laboratório de Química para a extração do amido das sementes.

\subsection{EXTRAÇÃO DO AMIDO}

Os frutos foram lavados em água potável, cortados ao meio com faca de aço inoxidável, separando as sementes da polpa. No final foram processados 50 frutos obtendo-se $360 \mathrm{~g}$ de sementes.

A moagem das sementes foi realizada em um moinho de facas (Tecnal, TE-625, Piracicaba, Brasil), provido de malha de $1 \mathrm{~mm}$, com uso abundante de água, para evitar o aquecimento. A fase leitosa foi separada da massa fibrosa usando panos alvejados. O amido contido na fase leitosa foi separado por peneiragens e sucessivas decantações usando peneiras de aço inoxidável (Bertel, Caieiras, Brasil) com malhas finas de 250 a $75 \mu \mathrm{m}$. O amido decantado foi filtrado a vácuo e desidratado em estufa com circulação de ar (Marconi, MA 033, Piracicaba, Brasil) a $45^{\circ} \mathrm{C}$, até massa constante $(\sim 12 \mathrm{~h})$. Após secagem, o amido foi reduzido, manualmente, a partículas finas e armazenado em vidro com tampa hermética. No final da extração se obteve, aproximadamente, $48 \mathrm{~g}$ de amido nativo (denominado de ASB).

\subsection{FOSFATAÇÃO DO AMIDO}

Com o intuito de alcançar níveis máximos de fosfatação no ASB, foi aplicado um delineamento experimental composto central. A fosfatação foi conduzido ao acaso em esquema fatorial $2^{2} \mathrm{com}$ três pontos centrais e três repetições. As variáveis independentes foram: concentração de ácido tripolifosfato de sódio (ATPS: 3, 5 e 7\%) e tempo de fosfatação (TF: 30, 40 e 50 min).

A Tabela 1 mostra os valores codificados e reais das variáveis independentes dos cinco níveis, e os tratamentos em relação à concentração de ATPS e TF. 
TABELA 1 - VALORES CODIFICADOS E REAIS DAS VARIÁVEIS APLICADAS NA

FOSFATAÇÃO DO AMIDO EXTRAÍDO DA SEMENTE DE SWARTZIA BURCHELLI.

\begin{tabular}{cccc}
\hline \multirow{2}{*}{ Variáveis } & \multicolumn{3}{c}{ Nível } \\
\cline { 2 - 5 } & -1 & 0 & 1 \\
\hline ATPS (\%) & 3 & 5 & 50 \\
q (min) & 30 & 40 & TF (min) \\
\hline Tratamento & ATPS (\%) & 30 \\
\hline T1 & 3 & 50 \\
T2 & 3 & 30 \\
T3 & 7 & 50 \\
T4 & 7 & 40 \\
T5 & 5 & 40 \\
T6 & 5 & 40 \\
T7 & 5 & \multicolumn{2}{c}{4}
\end{tabular}

A fosfatação foi realizada segundo o método descrito por Paschall (1964), com algumas adaptações. A proporção de ASB e ATPS foi de $3: 5(\mathrm{~m} / \mathrm{v})$. As misturas obtidas conforme a Tabela 1 foram agitadas em agitador mecânico pendular (Tecnal, TE-053, Piracicaba, Brasil) a 100 rpm, em tempos também descritos na Tabela $1 \mathrm{e}$, em seguida, filtradas a vácuo. $O$ material filtrado foi seco em estufa (Marconi, MA033, Piracicaba, Brasil) a $45^{\circ} \mathrm{C}$, por $48 \mathrm{~h}$; reduzido a partículas finas e novamente secado a $65^{\circ} \mathrm{C}$ até massa constante, lavado com $100 \mathrm{~mL}$ de etanol a $50 \%(\mathrm{v} / \mathrm{v})$. O produto resultante foi seco a $45{ }^{\circ} \mathrm{C}$ por $48 \mathrm{~h}$, seguido de diálise para remoção residual de sais de fósforo. A diálise foi realizada adicionando-se suspensões aquosas de amido fosfatado a $10 \%$ $(\mathrm{m} / \mathrm{v})$ em papel celofane e imersas em água destilada por sete dias, mantidas sob refrigeração em geladeira (aproximadamente a $6{ }^{\circ} \mathrm{C}$ ), sendo a água destilada diariamente trocada (LIMBERGER et al., 2008). Feita a diálise, o amido fosfatado foi seco a $45{ }^{\circ} \mathrm{C}$ e reduzido a partículas finas em peneiras de $150 \mu \mathrm{m}$, guardados em recipiente hermético até sua utilização. $O$ amido fosfatado assim obtido foi denominado de AFSB.

\subsection{CARACTERIZAÇÃO DOS AMIDOS NATIVO E FOSFATADOS}

\subsubsection{Composição Química}

As determinações dos teores de água, cinza, lipídio, proteína e fibra bruta dos amidos nativo e fosfatados foram realizadas segundo o método descrito pela Association of Official Analytical Chemists (AOAC, 1995). O teor de fósforo, $\mathrm{P}(\%)$, foi determinado de acordo com a técnica descrita pelo Instituto Adolfo Lutz (IAL, 2008) por espectrofotometria usando um espectrofotômetro UV/ visível (SP-2000UV) a $420 \mathrm{~nm}$. 


\subsubsection{Microscopia Óptica}

A forma e tamanho dos grânulos dos amidos foram analisados por meio de microfotografias realizadas segundo o método descrito por Vigneau et al. (2000), com algumas modificações. Uma quantidade pequena de amido foi imersa em álcool etílico e colocada sobre uma lâmina histológica recoberta com lamínula. A amostra foi observada e fotografada com microscópio óptico Leica (DMI, São Paulo) com aumento de 100x.

\subsubsection{Espectrocopia de Absorção na Região do Infravermelho}

As análises de espectroscopia de absorção na região do infravermelho com transformada de Fourier (FTIR) foram realizadas utilizando-se pastilhas de $\mathrm{KBr}$ e o equipamento Bomem Hartmann \& Braun/MB modelo 102, com condições de operação estabelecida: região $400-4000 \mathrm{~cm}^{-1}$, com 32 varreduras e resolução de $2 \mathrm{~cm}^{-1}$. Para o preparo das pastilhas, aproximadamente $1,0 \mathrm{mg}$ de cada amostra de amido foram misturados com $100 \mathrm{mg}$ de $\mathrm{KBr}$, sendo esta mistura, com baixa umidade, comprimida entre dois pastilhadores sob alta pressão (de aproximadamente oito toneladas) até a formação de uma pastilha translúcida e sem deformações. A análise foi realizada em duplicata.

\subsubsection{Difração de Raio-X}

A análise difração de raio-X foi realizada segundo o método descrito por Rindlav, Hulleman \& Gatenholm (1997). Os difratogramas dos amidos nativo e fosfatados foram obtidos utilizando o equipamento Rigaku (modelo RU 200 R, Osaca, Japão), em que, as amostras foram fixadas em suporte de alumínio e analisadas, à temperatura ambiente, operando com filtro monocromático, radiação Ka de cobre $(\lambda=1,54056 \AA)$, voltagem de $40 \mathrm{KV}$ e corrente de $50 \mathrm{~mA}$ e potência de 0,8 $\mathrm{kW}$; calibrado em relação à linha em $2 \theta=28,466^{\circ}$ do padrão de silício. $O$ comprimento de onda utilizado foi de $1,54 \AA$. As medidas foram feitas em varredura contínua no intervalo de $5^{\circ} \leq 2 \theta \leq 40^{\circ}$, sob velocidade de varredura de $2^{\circ}$ por minuto.

\subsubsection{Viscosidade de Pasta}

A viscosidade de pasta foi determinada segundo o manual do Rapid Visco Analyser (RVA). Suspensões de amido de $2,5 \mathrm{~g}$ em $25 \mathrm{~mL}$ de água, corrigidas para $14 \%$ de umidade, foram analisadas de acordo com o seguinte regime tempo/temperatura: $50^{\circ} \mathrm{C}$ por $1 \mathrm{~min}$, aquecimento de 50 a $95^{\circ} \mathrm{C}$ a uma taxa de $6{ }^{\circ} \mathrm{C} / \mathrm{min}$, manutenção da pasta a $95^{\circ} \mathrm{C}$ por 5 min e resfriamento de 95 a $50{ }^{\circ} \mathrm{C}$ a uma taxa de $6{ }^{\circ} \mathrm{C} / \mathrm{min}$.

A viscosidade de pasta foi expressa em cP (centpoise). Dos perfis gerados pelo RVA foram avaliadas a temperatura de empastamento (Te), viscosidade máxima (Vmax), quebra de viscosidade $\left(\mathrm{BD}\right.$, diferença entre a viscosidade máxima e a viscosidade mínima da pasta mantida a $95^{\circ} \mathrm{C}$ por 5 $\mathrm{min}$ ) e tendência à retrogradação (SB, diferença entre a viscosidade final e a viscosidade mínima a $95^{\circ} \mathrm{C}$ por $\left.5 \mathrm{~min}\right)$.

\subsection{6 Índice de Solubilidade em Água e Índice de Absorção de Água}

O índice de solubilidade em água (ISA) e o índice de absorção de água (IAA) foram determinados conforme os métodos descritos por Anderson (1982). 


\subsection{ANÁLISE ESTATÍSTICA}

As variáveis respostas foram os teores de fósforo, cinza, comprimento e largura do grânulo, cristalinidade, temperatura de pasta, viscosidade máxima, quebra de viscosidade, retrogradação, índice de solubilidade e índice de absorção de água.

Aplicou-se um modelo polinomial de segunda ordem (Equação 1) para estabelecer uma equação preditiva das variáveis resposta (KHURI \& CORNELL, 1987), em função de ATPS e TF. Sua adequação foi testada pela análise de variância e coeficiente de determinação ajustado $\left(R_{\text {aj }}^{2}\right)$.

$$
Y=b_{0}+\sum_{i=1}^{k} b_{i} X_{i}+\sum_{i=1}^{k} b_{i} X_{i}^{2}+\sum_{i<j 1}^{k} b_{j} X_{i} X_{j}+\varepsilon
$$

Em que, Y é a função resposta; $X$ é a variável codificada; b representa os coeficientes estimados pelos métodos dos mínimos quadrados; $\varepsilon$ é o erro experimental, sendo sua significância avaliada pelo valor da probabilidade $p$, adotando-se $p \leq 0,05$ para todos os ensaios, com o objetivo de avaliar quais coeficientes foram mais significativos e qual teve maior efeito.

Os gráficos de superfície de resposta foram gerados através do modelo matemático proposto nos níveis reais das variáveis, sendo a resposta em função do eixo Z; e os eixos $\mathrm{X}$ e $\mathrm{Y}$, as variáveis independentes.

Foi estudada a correlação de Pearson ( $r$ ) entre as variáveis respostas, a $5 \%$ de probabilidade, sendo considerada a correlação forte aquelas que tiveram valores de $\pm 0,6 \leq r< \pm 1$; moderada, $\pm 0,3$ $\leq r< \pm 0,6$; e fraca, $\pm 0,0 \leq r< \pm 0,3$ (CALLEGARI-JACQUES, 2003).

Como forma de comparação de resultados e agrupar as amostras de amido fosfatado com características semelhantes, foi utilizada a Análise de Componentes Principais (PCA).

A estatística e os gráficos de superfície de resposta foram obtidos por meio do programa Statistica for Windows versão 8.0 da StatSoft.

\section{RESULTADOS E DISCUSSÃO}

Durante a moagem das sementes da $S$. burchelli foi observada a formação de espuma branca, provavelmente, formada pela presença de esteróides ou de terpenos policíclicos (saponinas) que têm a propriedade de redução da tensão superficial da água e suas ações detergentes e emulsificante (SCHENKEL, GOSMANN \& ATHAYDE, 2001). Embora tenha dificultado a passagem da massa leitosa pelas peneiras, a espuma não interferiu na qualidade da extração de amido.

O rendimento em amido foi $9,8 \%$ em base úmida, considerado baixo quando comparado com de raízes de mandioca entre 21-33\% (MENDONÇA, MOURA \& CUNHA, 2003). Atribuiu-se esse baixo rendimento a fibras e outros constituintes da semente da $S$. burchelli, já que menor fração de componentes como fibras, proteínas e lipídios na matéria-prima pode proporcionar maiores rendimentos (FRANCO et al., 2001).

O ASB apresentou baixos teores de cinza $(0,02 \%)$, proteína $(0,24 \%)$, lipídio $(0,04 \%)$, fibra bruta $(0,03 \%)$, traços de fósforo $(0,001 \%)$ e teor de água $(5,52 \%)$, indicando que o amido extraído é de boa qualidade por apresentar menor conteúdo desses componentes.

Os teores de fibra, lipídio e proteína dos AFSB não variaram após fosfatação. No entanto, o teor de fósforo aumentou até aproximadamente $0,27 \%$ (Figura $1 \mathrm{~A}$ ) acarretando o aumento de cinza até $0,32 \%$ (Figura 1B). Estes teores correlacionaram positivamente entre si ( $r=0,99 ; p<0,001)$; reforçando às observações feitas por Limberger et al. (2008) e Pereira e Ascheri (2011), referindo que esse comportamento se deve à incorporação de grupos fosfatos na estrutura do amido. 

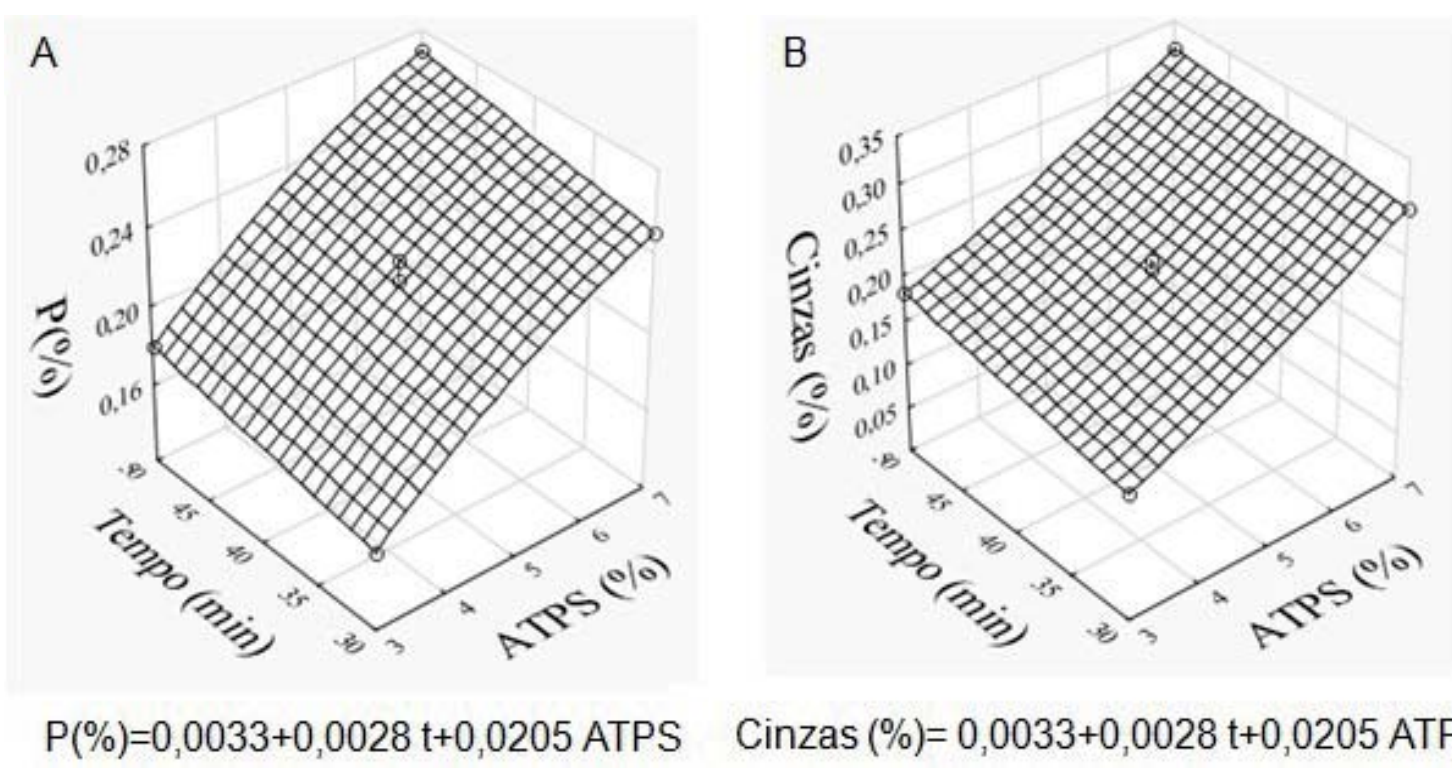

$P(\%)=0,0033+0,0028 t+0,0205$ ATPS Cinzas $(\%)=0,0033+0,0028 t+0,0205$ ATPS

\author{
FIGURA 1 - VARIAÇÃO DOS TEORES DE FÓSFORO E CINZA DOS AMIDOS \\ FOSFATADOS DE S. BURCHELLI EM FUNÇÃO DA CONCENTRAÇÃO DE ÁCIDO \\ TRIPOLIFOSFATO DE SÓDIO (ATPS) E TEMPO DE FOSFATAÇÃO. A) TEOR DE \\ FÓSFORO E B) TEOR DE CINZAS.
}

Os amidos fosfatados apresentaram valores percentuais de fósforo e cinza crescentes conforme o aumento da concentração de ATPS e TF. Todos esses amidos tiveram teor de fósforo dentro do permitido pela legislação para uso alimentar, menor que $0,4 \%$ segundo a Food and Drug Administration (2007). O amido tratado com ATPS a 7\% por $50 \mathrm{~min}$ apresentou maior teor de fósforo $(0,32 \%)$. Esses resultados indicam os amidos fosfatados com maior quantidade de ATPS em maiores tempos de fosfatação apresentarão maior teor de fósforo.

Com o FTIR (Figura 2) não foi possível observar diferenças entre si nas bandas de absorção, havendo apenas pequenas mudanças em suas transmitâncias segundo o teor de fósforo presente no amido. As observações de alterações nos espectros de amidos fosfatados foi dificultada devido a sobreposição de amplas bandas de backbone de amido, fato também verificado por Passauer, Liebner \& Fischer (2006). Contudo, a máxima absorção observada na banda arredondada (entre $3600-3200$ ) foi $3461 \mathrm{~cm}^{-1}$ que corresponde a grupos hidroxilas da D-glicose presente no amido (WANG et al., 2009). Os picos que caracterizam as ligações $\mathrm{C}-\mathrm{H}$ do anel e do estiramento da ligação glicosídica C-O apresentaram valores de número de onda de máxima absorção em 2926 e $1644 \mathrm{~cm}^{-1}$, respectivamente, próximos aos encontrados por Nakason et al. (2001) e Wang et al. (2009) para C-H entre 2923-2926 $\mathrm{cm}^{-1}$, e Silverstein \& Webster (2000) e Sandler et al. (1998) para $\mathrm{C}-\mathrm{O}$ de $1650 \mathrm{~cm}^{-1}$. As bandas espectrais de máxima absorbância de 1162; $1086 \mathrm{e} 1014 \mathrm{~cm}^{-1}$ indicam a presença das ligações C-O-C. Nakason et al. (2001), em amido de mandioca detectaram esses picos nas bandas espectrais de máxima absorbância de 1156, 1051 e $1023 \mathrm{~cm}^{-1}$, respectivamente. 

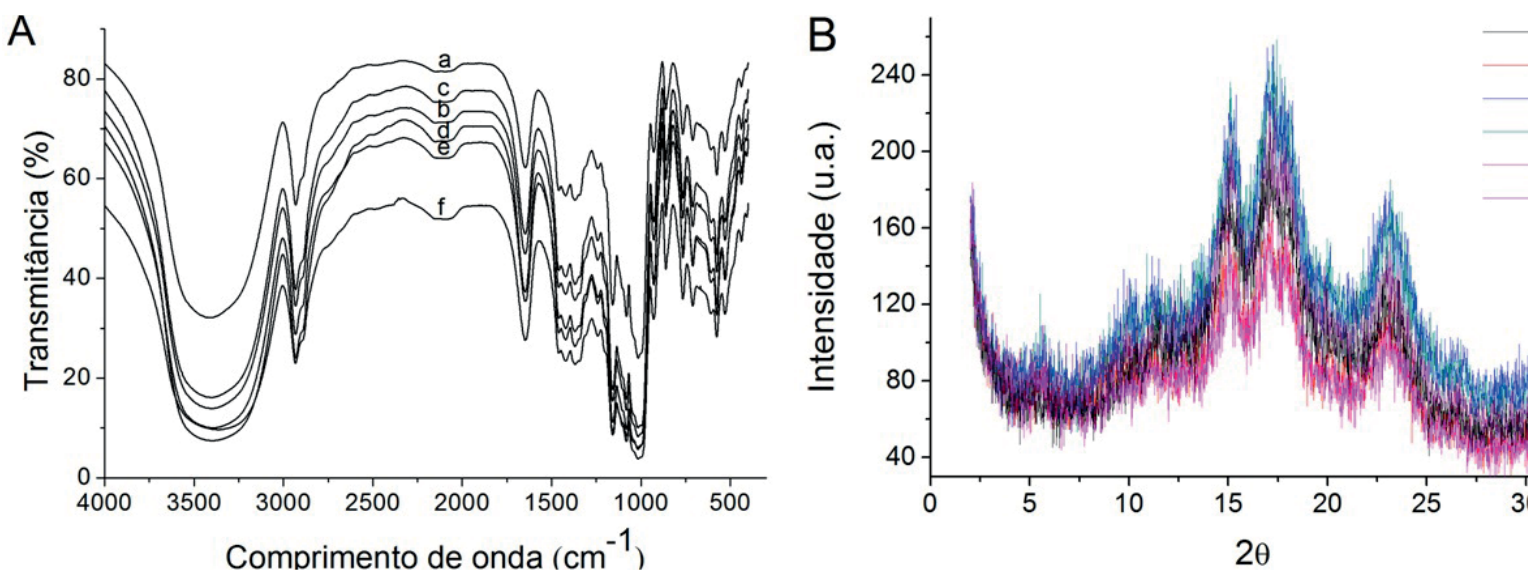

\section{FIGURA 2 - ESPECTROS DE FTIR E DE RAIOS-X DOS AMIDOS FOSFATADOS DE SWARTZIA BURCHELLI EM FUNÇÃO DA CONCENTRAÇÃO DE ÁCIDO} TRIPOLIFOSFATO DE SÓDIO (ATPS) E DO TEMPO DE FOSFATAÇÃO. A) NATIVO, B) 3\%, $30 \mathrm{MIN}$, C) $3 \%, 50 \mathrm{MIN}$, D) $7 \%, 30 \mathrm{MIN}$, E) $7 \%, 50 \mathrm{MIN}$, F) $5 \%, 40$ MIN DE ATPS E TEMPO DE FOSFATAÇÃO, RESPECTIVAMENTE.

Segundo Stuart (2004), os compostos alifáticos de fósforo contendo ligação P-O-C deveriam mostrar uma banda forte entre 1050 e $950 \mathrm{~cm}^{-1}$; já a presença de grupos $\mathrm{P}=\mathrm{O}$ deve ser evidenciada por bandas em 1260-1240 $\mathrm{cm}^{-1}$. Essas bandas são imperceptíveis na Figura 2A entre 1050 e $930 \mathrm{~cm}^{-1}$ para $\mathrm{P}-\mathrm{O}-\mathrm{C}$ e $1245 \mathrm{~cm}^{-1}$ para $\mathrm{P}=\mathrm{O}$. Com ressonância magnética nuclear poderiam ser detectados esse grupos fosfato.

Os espectros de raios-X (Figura $2 \mathrm{~B}$ ) não diferem quanto a seu padrão de cristalinidade. Todos eles apresentaram picos principais em 15,$2 ; 17,3 ; 18,3$ e $23,2^{\circ}$, característicos de amidos contendo padrão de cristalinidade do tipo A, também visto em amidos de semente de manga e milho por Sandhu \& Lim (2008) e milho e trigo por Lima et al. (2012). Entretanto, nos espectros dos amidos fosfatados se observam picos mais estreitos, intensos e finos que do amido nativo. Segundo Neto (2003), esses tipos de espectro são típicos de amidos que contém maior número de regiões cristalinas na estrutura ramificada da amilopectina, o que infere que o grau de cristalinidade do amido nativo aumentou com o aumento de grupos fosfato que agem reforçando as cadeias laterais da amilopectina.

A Figura 3 mostra os gráficos de superfície de resposta dos valores de grau de cristalinidade Figura $3 \mathrm{~A}$ ), comprimento (Figura $3 \mathrm{~B}$ ) e largura (Figura $3 \mathrm{C}$ ) dos grânulos de amidos nativo e fosfatados. De acordo com a Figura 3A, o grau de cristalinidade dos amidos fosfatados aumentou com o incremento dos níveis das variáveis ATPS e TF. Esse aumento corresponde 4 a $29 \%$ da cristalinidade do amido nativo, sendo o maior percentual observado no amido que foi fosfatado por 50 min com $7 \%$ de ATPS (grau de cristalinidade de 55,3\%).

Quanto ao tamanho, ASB apresentou menor comprimento $(\sim 18 \mu \mathrm{m})$ e largura, $(\sim 16 \mu \mathrm{m})$ em relação aos amidos fosfatados com comprimento e largura de 18,5-22,6 $\mu \mathrm{m}$ e 16,5-18,5 $\mu \mathrm{m}$, respectivamente. Essas medidas correlacionaram positivamente com o teor de fósforo, sendo $r=$ $0,93(p<0,01)$ para comprimento e $r=0,87(p=0,01)$ para largura, indicando que o aumento das dimensões desses grânulos se deve aos efeitos causados pela combinação das variáveis ATPS e TF. 

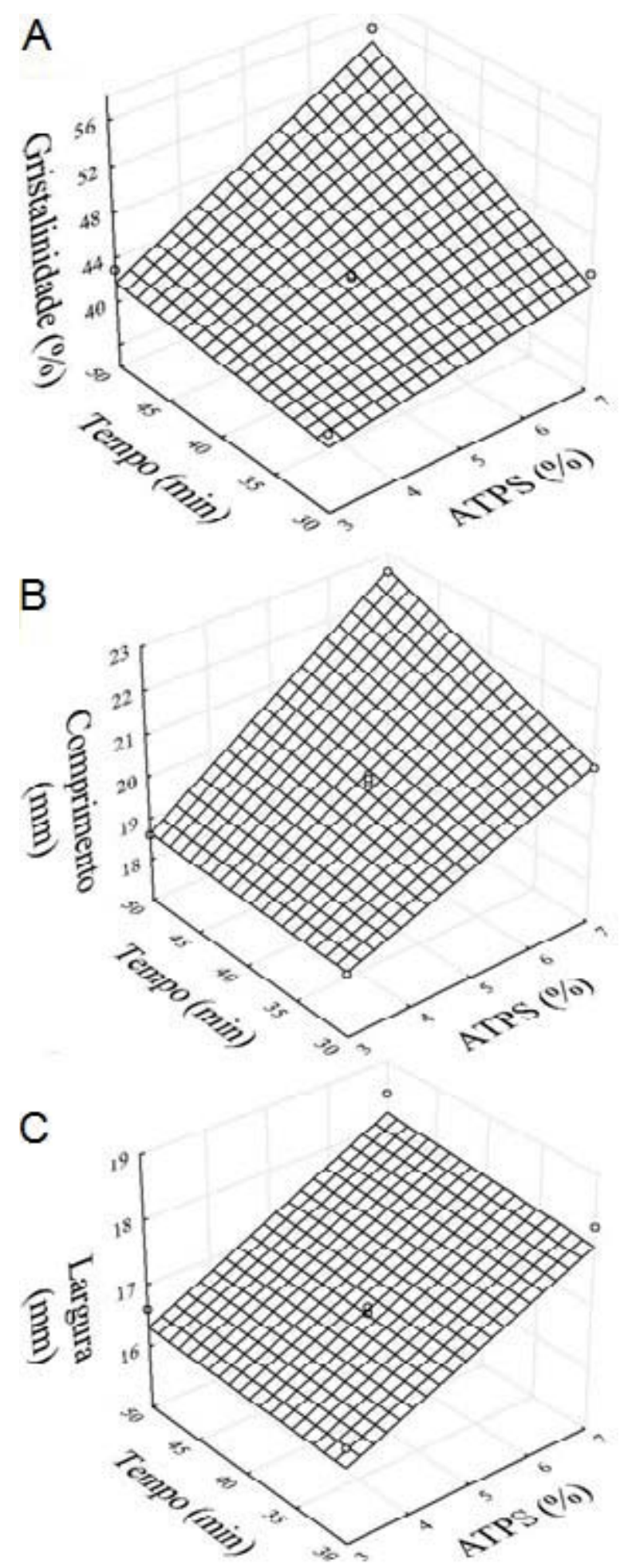

FIGURA 3 - VARIAÇÃO DO GRAU DE CRISTALINIDADE E TAMANHO DOS GRÂNULOS DE AMIDOS NATIVO E FOSFATADOS DE SWARTZIA BURCHELLI EM FUNÇÃO DA CONCENTRAÇÃO DE ÁCIDO TRIPOLIFOSFATO DE SÓDIO (ATPS) E TEMPOS DE FOSFATAÇÃO. A) CRISTALINIDADE, B) COMPRIMENTO E C) LARGURA. 
As imagens microscópicas da Figura 4 mostram que os grânulos do ASB apresentaram superfície lisa com diferentes tamanhos e formas. Dentre as formas são observadas grânulos arredondados, elípticos, truncadas e irregulares. Além dessas formas, nos amidos fosfatados se observam grânulos inchados e danificados, significando que o amido nativo sofre danos aparentes após fosfatação o que pode influenciar nas propriedades de pasta do amido.
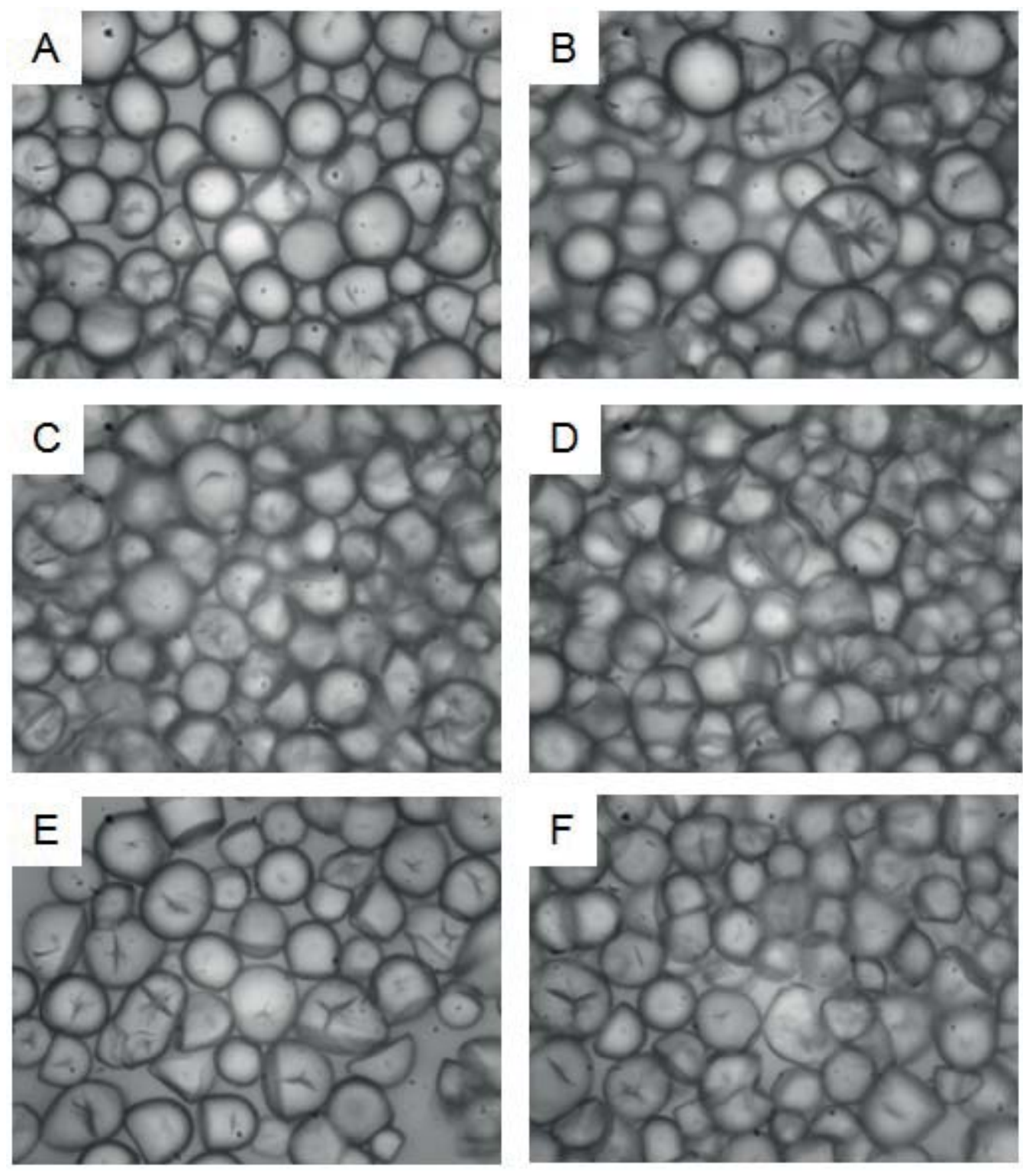

\section{FIGURA 4 - MICROFOTOGRAFIAS DOS GRÂNULOS DE AMIDOS NATIVO E FOSFATADOS DE SWARTZIA BURCHELLI EM FUNÇÃO DA CONCENTRAÇÃO DE ÁCIDO TRIPOLIFOSFATO DE SÓDIO EM DIFERENTES TEMPOS DE FOSFATAÇÃO (AUMENTO DE 100X). A) NATIVO. B) 3\%, 30 MIN. C) 3\%, 50 MIN. D) $7 \%, 30$ MIN. E) 7\%, 50 MIN. F) 5\%, 40 MIN.}

Após fosfatação, a temperatura de pasta do amido da S. burchelli diminuiu de $83,8^{\circ} \mathrm{C}$ até aproximadamente $78{ }^{\circ} \mathrm{C}$, sendo o amido fosfatado por $30 \mathrm{~min}$ com $3 \%$ de ATPS que apresentou a menor temperatura de $77,9^{\circ} \mathrm{C}$ e a maior temperatura de pasta $\left(79,1^{\circ} \mathrm{C}\right)$ foi detectada no amido 
tratado com $7 \%$ de ATPS por 50 min de fosfatação.

$O$ pico de viscosidade, quebra de viscosidade e tendência à retrogradação do ASB foram afetados pelos parâmetros do processo de fosfatação (Figura $5 \mathrm{~A}$ a $5 \mathrm{C}$, respectivamente). $\mathrm{O}$ pico de viscosidade dos amidos fosfatados detectados entre 2728-3058 cP diminuiu entre 31 a $39 \%$ com relação a ASB que apresentou pico aproximadamente de $4441 \mathrm{cP})$. Essa propriedade de pasta correlacionou negativamente com o teor de fósforo contidos nos amidos fosfatados $(r=-0,96, p<$ 0,01 ). Esse comportamento reológico é típico de amidos com forte estrutura cristalina (padrão de cristalinidade tipo A) como ocorre em amidos de cereais que formam pastas com baixo pico de viscosidade (CHUNG et al., 2008).

A diminuição da viscosidade dos amidos fosfatados indica maior interação entre os componentes dos grânulos na presença dos grupos fosfato (BATISTA et al. 2010), reforçando a região amorfa (KARIM et al. 2007) da amilopectina que contribui para o intumescimento do grânulo. Esse comportamento provavelmente se deve também ao fato que os amidos fosfatados apresentam maior quantidade de grânulos danificados que deixaram de intumescer durante o aquecimento no RVA, já que quanto menor é o intumescimento dos grânulos maior a quantidade de água livre associada à diminuição da viscosidade das pastas de amido (MING et al., 1997).

Ao contrário do ASB (com BD= 2377,2 cP), os amidos fosfatados (com 828,5 $\square \mathrm{BD} \square$ 951,9 $\mathrm{CP}$ ) apresentaram menores valores de quebra de viscosidade, diminuindo com o aumentos dos níveis de concentração de ATPS nos diferentes tempos de fosfatação (Figura 5B). Esta observação também indica que a fosfatação reforçou a estrutura granular do amido. BD correlacionou com PV $(r=0,77, p=0,04)$. Chan, Bhat $\&$ Karim (2010) referem que a evidente quebra de viscosidade observada em amidos modificados em comparação dos amidos nativos provavelmente se deve à estrutura enfraquecida dos grânulos durante o processo de modificação química, facilitando a quebra da estrutura granular. Entretanto, no presente estudo observou-se que a fosfatação tornou os grânulos de amido mais resistentes e estáveis ao aquecimento e cisalhamento.

Por apresentar grânulos grandes de amido e muito deles danificados, esperava-se dos amidos fosfatados alta retrogradação como resultados do esfriamento de suas pastas durante o processo do RVA, já que, segundo Yuan et al. (2007) maiores valores de SB são encontrados em amidos que contêm grânulos grandes, devido à sua maior fragilidade. No entanto, estes amidos fosfatados apresentam maior estabilidade à retrogradação $(1.247-1.600,5 \mathrm{cP})$ que o ASB $(1.740$ cP). SB não correlacionou com o tamanho dos grânulos ( $r=-0,8739, p=0,126)$ e nem com o grau de cristalinidades ( $r=-0,9272, p=0,073$ ), por isso, atribuí-se a diminuição dessa propriedades de pasta, à natureza da estrutura molecular da amilopectina, pois, ela é responsável pela retrogradação dos amidos.

O ISA e o IAA dos amidos fosfatados aumentaram com o aumento dos níveis da concentração de ATPS e tempo de fosfatação (Figuras 6A e 6B, respectivamente). $O$ amido absorve determinada quantidade de água a partir da sua região amorfa, porém ele não se solubiliza em água fria como pode ser verificado no ASB que apresentou $0,22 \%$ de solubilidade. Enquanto que, em virtude da danificação do amido pelo processo de fosfatação, a amilose ficou exposta à hidratação, por isso os amidos fosfatados ficaram mais solúveis (0,37-0,65\%). $O$ índice de absorção de água desses amidos (1,96-2,07 $\left.\mathrm{g} \mathrm{g}^{-1}\right)$ foi maior que a do ASB (1,94 $\left.\mathrm{g} \mathrm{g}^{-1}\right)$. Embora esses índices não correlacionem com o teor de fósforo $(r<0,40, p>0,37)$, a absorção de água dos amidos fosfatados a temperatura ambiente ocorre porque os grupos fosfato, negativamente carregados, introduzidos nas cadeias de amido promovem uma redução nas forças intermoleculares de ligação, devido à repulsão entre si (SITOHY et al., 2000, LAWAL, 2004, BATISTA et al., 2010). 

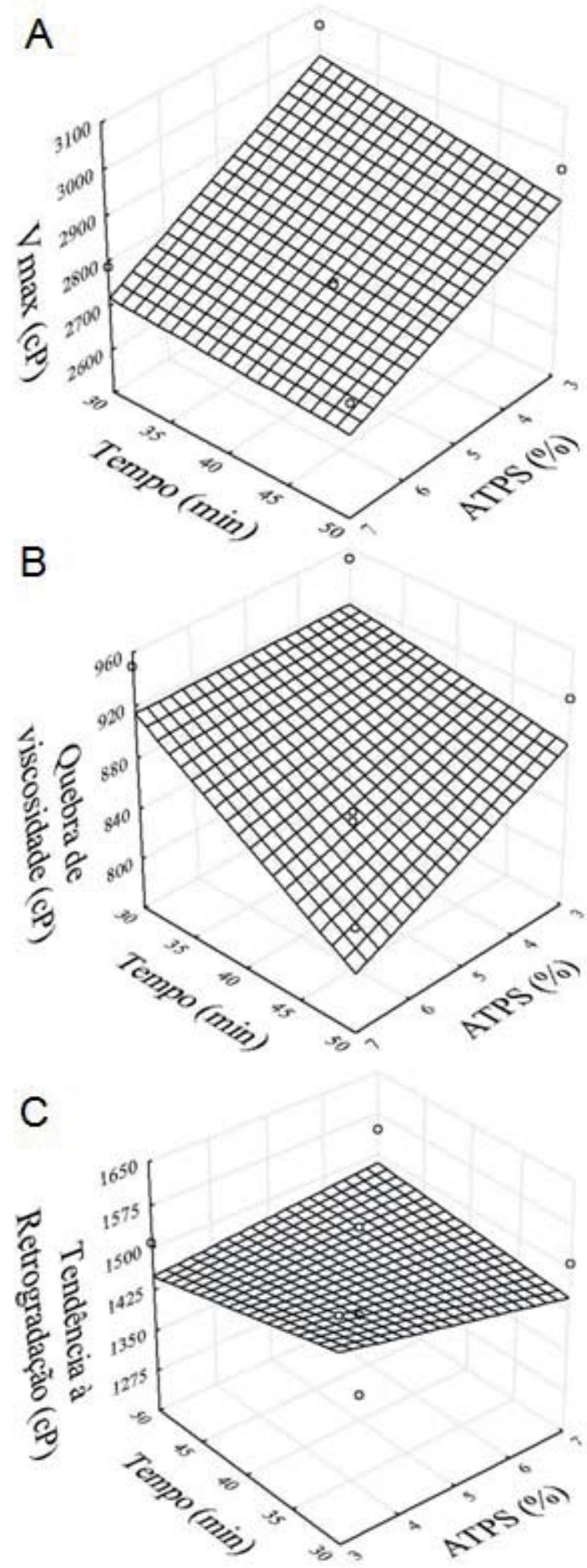

FIGURA 5 - VARIAÇÃO DAS PROPRIEDADES DE PASTA DOS AMIDOS FOSFATADOS DE SWARTZIA BURCHELLI EM FUNÇÃO DA CONCENTRAÇÃO DE ÁCIDO TRIPOLIFOSFATO DE SÓDIO (ATPS) E TEMPO DE FOSFATAÇÃO. A) VISCOSIDADE MÁXIMA (VMAX), B) QUEBRA DE VISCOSIDADE E C) TENDÊNCIA À RETROGRADAÇÃO. 

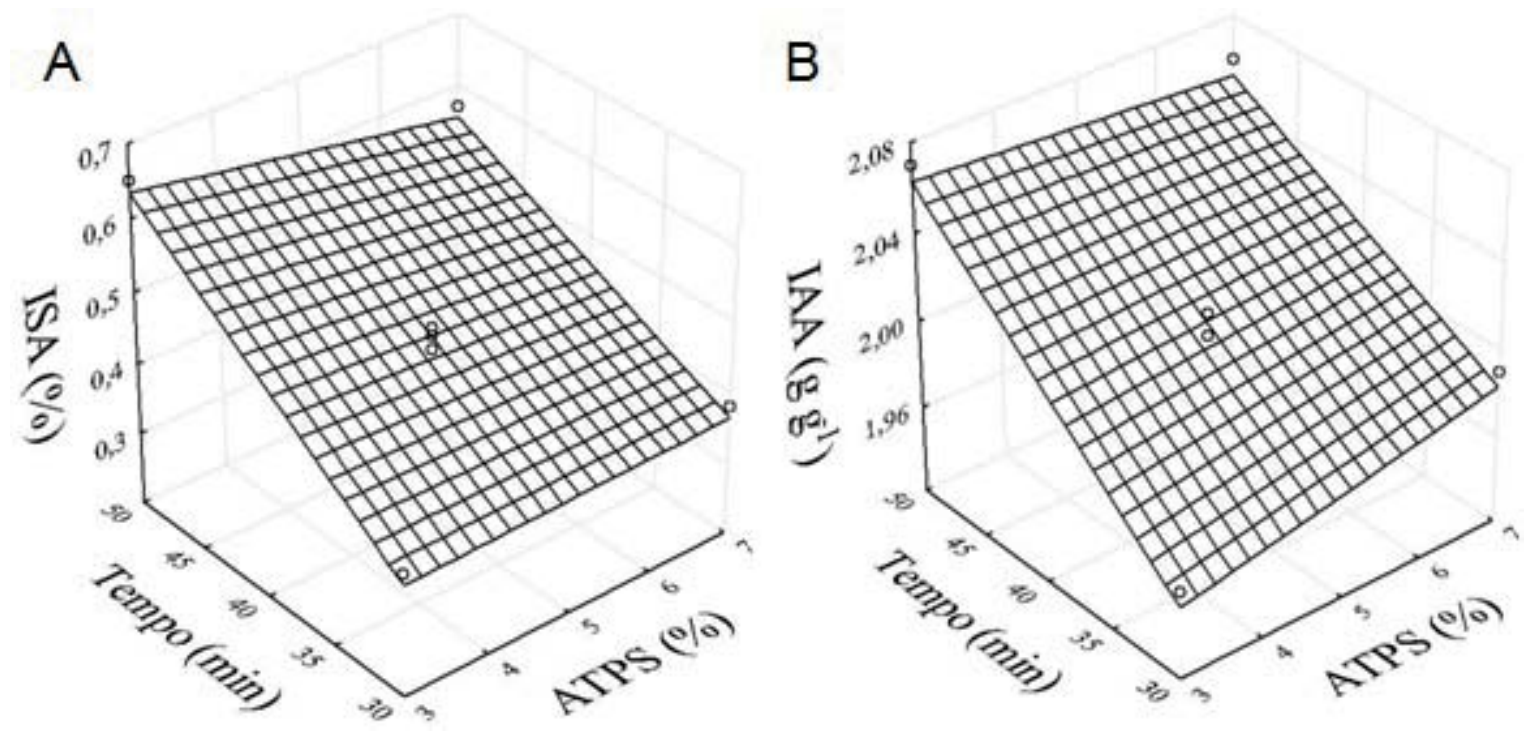

\section{FIGURA 6 - VARIAÇÃO DOS ÍNDICES DE SOLUBILIDADE EM ÁGUA (ISA) E ABSORÇÃO DE ÁGUA (IAA) DE AMIDOS FOSFATADOS DE SWARTZIA BURCHELLI EM FUNÇÃO DA CONCENTRAÇÃO DE ÁCIDO TRIPOLIFOSFATO DE SÓDIO (ATPS) E TEMPO FOSFATAÇÃO. (A) ISA, B) IAA.}

Visando agrupar os tratamentos semelhantes, foi utilizada a análise de componentes principais (ACP) para analisar um pequeno número de combinações lineares, os componentes principais $(\mathrm{CP})$, de um conjunto de variáveis que detém o máximo de informações possíveis (MUTIHAC \& MUTIHAC, 2008).

Na representação gráfica da ACP, Figura 7, cada eixo explica uma porcentagem da variação total que existe entre as amostras. O primeiro eixo explica a maior parte da variabilidade entre as amostras $\left(\mathrm{CP}_{1}=75,28 \%\right)$ e o segundo eixo $\left(\mathrm{CP}_{2}\right)$ explica $19,73 \%$ da variação. Juntos representam $95,01 \%$ da variação dos resultados. O restante da variação (4,99\%) foi devido a outros componentes principais que não foram apresentados pelo fato de os dois primeiros explicarem mais de $80 \%$ da variação dos resultados (YASUMURA, D'ALMEIDA \& PARK, 2012).

AFigura 7A mostra o gráfico das projeções dos objetos originais no espaço das componentes principais, ou seja, das novas coordenadas dos objetos nas novas variáveis que são as CPs. Quando os escores estão próximos entre si, significa que são similares em relação aos atributos julgados, já quando distantes uns dos outros, apresentam grandes diferenças. Sendo assim, nota-se que existem quatro grupos com padrões distintos. 

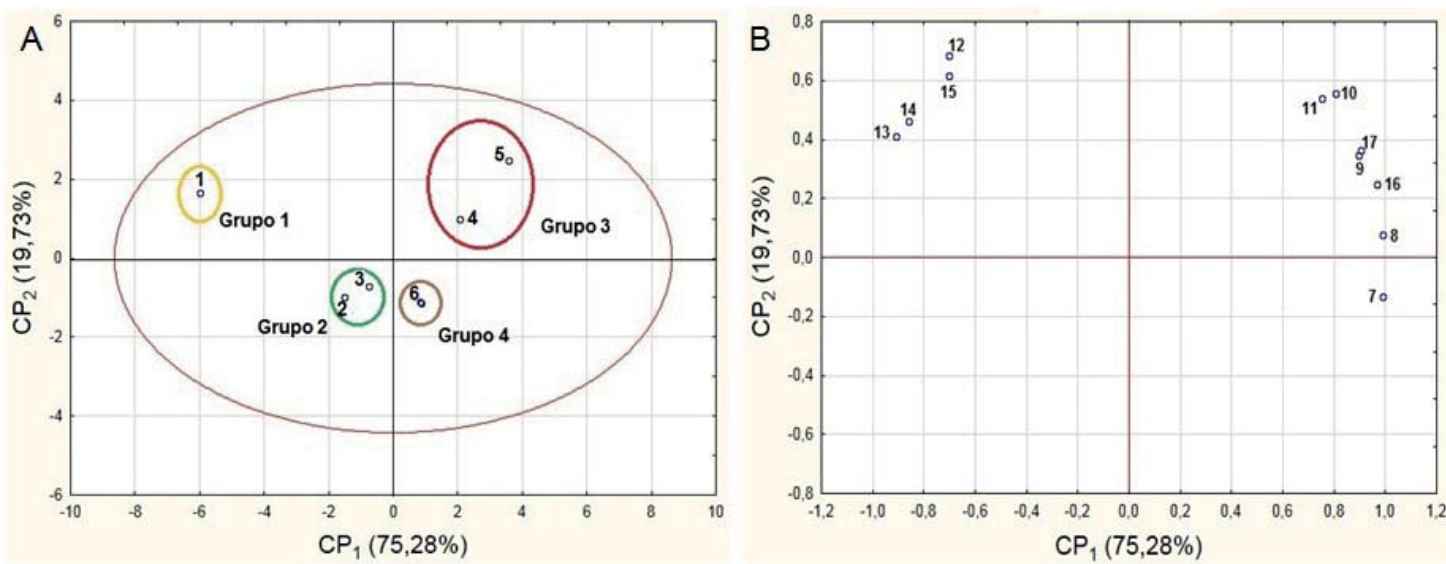

FIGURA 7 - ANÁLISE DE COMPONENTES PRINCIPAIS $\left(\mathrm{CP}_{1} \mathrm{E} \mathrm{CP}_{2}\right)$ DOS AMIDOS NATIVO E FOSFATADOS DE SWARTZIA BURCHELLI COM ÁCIDO TRIPOLIFOSFATO DE SÓDIO

EM DIFERENTES TEMPOS DE AGITAÇÃO. A) ANÁLISE DOS TRATAMENTOS E B)

ANÁLISE DE VARIÁVEIS. 1) AMIDO NATIVO. AMIDOS FOSFATADOS: 2) 3\%, 30 MIN, 3) $3 \%, 50 \mathrm{MIN}, 4) 7 \%, 30 \mathrm{MIN}, 5) 7 \%, 50 \mathrm{MIN}, 6) 5 \%, 30 \mathrm{MIN}, 7)$ TEOR DE FÓSFORO, 8) TEOR

DE CINZAS, 9) COMPRIMENTO, 10) LARGURA, 11) GRAU DE CRISTALINIDADE, 12)

TEMPERATURA DE PASTA, 13) VISCOSIDADE MÁXIMA, 14) QUEBRA DE VISCOSIDADE, 15) TENDÊNCIA À RETRGRADAÇÃO, 16) ÍNDICE DE SOLUBILIDADE EM ÁGUA, 17) ÍNDICE DE ABSORÇÃO DE ÁGUA.

Os atributos julgados foram teor de fósforo e cinzas, comprimento, largura, cristalinidade, temperatura de pasta, viscosidade máxima, quebra de viscosidade, tendência à retrogradação, índice de solubilidade em água e índice de absorção de água. O primeiro grupo é formado pelo amido nativo, já os outros grupos referem-se aos amidos fosfatados, sendo o segundo formado pelos amidos submetidos à $3 \%$ de ATPS, o terceiro à $7 \%$ de ATPS e o quarto à $5 \%$ de ATPS. De modo geral, observa-se que os amidos com mesma concentração de ATPS apresentaram distribuição mais uniforme (Figura 7A), e que o teor de fósforo foi determinante para os resultados de todas as variáveis, visto que, foi observada uma relação proporcional ou inversamente proporcional aos demais atributos (Figura 7B).

\section{CONCLUSÕES}

As sementes da Swartzia burchelli apresentam baixo rendimento em amido ( 10\%), porém de fácil extração e purificação (99.7\% de pureza). O amido fosfatado da $S$. burchelli apresentam propriedades diferenciadas principalmente a baixa tendência à retrogradação e alta viscosidade de pasta $( \pm 3000 \mathrm{cP})$. A baixa temperaturas de gelatinização $\left(78^{\circ} \mathrm{C}\right)$ implica, que em condições de processos industriais, não haveria necessidade de maiores valores de consumo de energia para a formação de pasta. Por apresentar menor retrogradação e maior transmitância, os amidos fosfatados da S. burchelli podem ser empregados para a elaboração de alimentos que requerem baixo grau de sinerese, principalmente de alimentos sujeitos a baixas temperaturas de armazenagem, bem como para seu uso em filmes e coberturas comestíveis. O amido fosfatado por 40 min com $5 \%$ de ATPS apresentou menor retrogradação e quebra de viscosidade e maior transmitância; enquanto que o amido fosfatado por 50 min com $7 \%$ de ATPS apresentou maior teor de cinzas e fósforo, grau de 
cristalinidade, comprimento e largura de seus grânulos e maior temperatura de pasta.

\section{ABSTRACT \\ STARCH PHOSPHATE OF THE SWARTZIA BURCHELLI: PHYSICAL AND PHYSICO- CHEMICAL PROPERTIES}

Swartzia burchelli is a Leguminosae that occurs in the Brazilian Cerrado biome, known as "Fat-of-chicken." The starch contained in its seeds can be availed for various technological purposes, however, requires modification for your specific use. The aim of this work was to study the characteristics of phosphate starch $\mathrm{S}$. burchelli depending on the concentration of sodium tripolyphosphate (3,5 and 7\%) and acid phosphate of time (30, 40 and $50 \mathrm{~min})$. The experiment was conducted in a factorial $2^{2}$ design with three center points, analyzed using Response Surface Methodology. Starches were determined their chemical composition, phosphorus and ash, shape and size, X-ray diffraction, infrared spectroscopy, pulp viscosity index and water solubility and water absorption. The appropriateness of a second-order polynomial model was tested by analysis of variance to establish a predictive equation of the experimental data. Responses were also analyzed by applying Pearson correlation and principal component analysis. Different size, degree of crystallinity, gelatinisation and pasting, water solubility and water absorption were observed due to the nature of the native starch or phosphating process. The starch phosphate for 40 min with $5 \%$ ATPS and showed less retrogradation viscosity and higher breaking transmittance; whereas the starch phosphate for 50 min with 7\% ATPS showed a higher content of ash and phosphorus, degree of crystallinity, its length and width, and beads folder higher temperature.

KEYWORDS: FRUIT OF THE CERRADO; RAPID VISCOSITY ANALYZER; RAIOS-X; SWELLING POWER, WATER SOLUBILITY; RESPONSE SURFACE METHODOLOGY.

\section{REFERÊNCIAS}

1 ANDERSON, R. A. Water absorption and solubility and amylograph characteristics of roll-cooked small grain products. Cereal Chemistry, v.59, n.4, p.265-269, 1982.

2 AOAC. Official methods of analysis of the Association of the Official Analysis Chemists. 16.ed. Arlington: Association of Analytical Communities, 1995.

3 ASCHERI, D. P. R.; MOURA, W. S.; ASCHERI, J. L. R.; CARVALHO, C. W. P. Caracterização física e físico-química de rizomas e amido do lírio-do-brejo (Hedychium coronarium). Pesquisa Agropecuária Tropical, v.40, n.2, p.159-166, 2010.

4 BATISTA, W. P.; SILVA, C. E. M.; LIBERATO, M. C. Propriedades químicas e de pasta dos amidos de trigo e milho fosforilados. Ciência e Tecnologia de Alimentos, v.30, n.1, p.88-93, 2010.

5 BOURTOOM, T. Edible films and coatings: characteristics and properties. International Food Research Journal, v.15, n.3, p.237-248, 2008.

6 CALLEGARI-JACQUES, S. M. Bioestatística: princípios e aplicações. Porto Alegre: Artmed, 2003. 365 p.

7 CEREDA, M. P. Propriedades gerais do amido. São Paulo: Fundação Cargill, 2002. 221 p.

8 CHAN, H. T.; BHAT, R.; KARIM, A. A. Effects of sodium dodecyl sulphate and sonication treatment on physicochemical properties of starch. Food Chemistry, v.120, n.3, p.703-709, 2010.

9 CHUNG, H. J.; LIU, Q.; HOOVER, R.; WARKENTIN, T. D.; VANDENBERG, B. In vitro starch digestibility, expected glycemic index, and thermal and pasting properties of flours from pea, lentil and chickpea cultivars. Food Chemistry, v.111, n.2, p.316-321, 2008. 
10 DHANAPAL, A.; SASIKALA P.; RAJAMANI, L.; KAVITHA V.; YAZHINI. G.; BANU, M. S. Edible films from Polysaccharides. Food Science and Quality Management, v.3, s/n, p.9-17, 2012.

11 FOOD AND DRUG ADMINISTRATION. Food starch modified: code of federal regulation. Washington: FDA, 2007.

12 FRANCO, C. M. L.; DAIÚTO, E. R.; DEMIATE, I. M.; CARVALHO, L. J. C. B.; LEONEL, M.; CEREDA, M. P.; VILPOUX, O.; SARMENTO, S. B. S. Metodologia de análise se amido. In: Propriedades gerais do amido. São Paulo: Fundação Cargill, 2002. p.184-203.

13 HERNÁNDEZ-MEDINA, M.; TORRUCO-UCO, J.G.; CHEL-GUERRERO, L.; BETANCUR-ANCONA, D. Caracterización fisicoquímica de almidones de tubérculos cultivados en Yucatán, México. Ciência e Tecnologia de Alimentos, v.28, n.3, p.718-726, 2008.

14 IAL. Métodos físico-químicos para análise de alimentos. 4ed., São Paulo: Instituto Adolfo Lutz, 2008.

15 KARIM, A. A.; TOON, L. C.; LEE, V. P.; ONG, W. Y.; FAZILAH, A.; NODA T. Effects of phosphorus contents on the gelatinization and retrogradation of potato starch. Journal of Food Science, v.72; n.2, p.132-138, 2007.

16 KASIRAJAN, S.; NGOUAJIO, M. Polyethylene and biodegradable mulches for agricultural applications: A review. Agronomy for Sustainable Development, v.32, n.2, p.501-529, 2012.

17 KHURI, A. I.; CORNELL, J. A. Response Surfaces, New York: Marcel Dekker, 1987.

18 LATNER, K.; O'KRAY, C.; JIANG, J. China, peoples republic of bio-fuels ASB alternative future for agriculture 2006. Washington: USDA Foreign Agricultural Service, 2006. GAIN Report Number: CH6049, 14p. Disponível em: <http://apps. fas.usda.gov/gainfiles/200608/146208611.pdf>. Acesso em: 29/06/2014.

19 LAWAL, O. S. Succinyl and acetyl starch derivatives of a hybrid maize: Physicochemical characteristics and retrogradation properties monitored by differential scanning calorimetry. Carbohydrate Research, v.339, n.16, p.2673-2682, 2004.

20 LEONEL, M. Análise da forma e tamanho de grânulos de amidos de diferentes fontes botânica. Ciência e Tecnologia de Alimentos, v.27, n.3, p.579-588, 2007.

21 LIMA, N. B. B; CABRAL, T. B.; NETO, R. P. C.; TAVARES, M. I. B.; PIERUCCI, A. P. T. Estudo do amido de farinhas convencionais comestíveis. Polímeros, v.22, n.5, p.486-490, 2012.

22 LIMBERGER, V. M.; SILVA, L. P.; EMANUELLI, T.; COMARELA, C. G.; PATIAS, L. D. Modificação química e física do amido de quirera de arroz para aproveitamento na indústria de alimentos. Química Nova, v.31, n.1, p.84-88, 2008.

23 LINDEBOOM, N.; CHANG, P. R.; TYLER, R. T. Analytical, biochemical and physicochemical aspects of starch granule size, with emphasis on small granule starches: a review. Starch/Stärke, v.56, n.3-4, p.89-99, 2004.

24 MANSANO, V. F.; LEWIS, G. P.; TOZZI, A. M. G. A. Swartzia burchelli (Leguminosae- Papilionoideae) from Goiás, Brazil; a new name for a taxon proposed by Bentham. Kew Bull, v.57, n.3, p.635-638, 2002.

25 MENDONÇA, H. A.; MOURA, G. M.; CUNHA, E. T. Avaliação de genótipos de mandioca em diferentes épocas de colheita no Estado do Acre. Pesquisa Agropecuária Brasileira, v.38, n.6, p.1-8, 2003.

26 MING, Z.; MORRIS, C. F.; BATEY, I. L.; WRIGLEY, C. W. Sources of variation for starch gelatinization, pasting, and gelation properties in wheat. Cereal Chemical, v.74, n.1, p.63-71, 1997.

27 MOJOVIĆ, L.; NIKOLIĆ, S.; RAKIN, M.; VUKASINOVIĆ, M. Production of bioethanol from corn meal hydrolyzates, Fuel, v.85, n.12-13, p.1720-1755, 2006.

28 MUTIHAC, L.; MUTIHAC, R. Mining in chemometrics. Analytica Chimica Acta, v.612, n.1, p.1-18, 2008.

29 NAKASON, C.; KAESMAN, A.; HOMSIN, S.; KIATMJORWONG, S. Rheological and curing behavior of reactive blending. I. Maleated natural rubber-cassava starch. Journal of Applied Polymer Science, v.81, n.11, p.2803-2813, 2001.

30 NETO, R. B. Raio-X. In. CANEVAROLO JR., S.V. Técnicas de Caracterização de polímeros. São Paulo: Artiber Editora, 2003. p.41-60.

31 NODA, T.; KOTTEARACHCHI, N. S.; TSUDA, S.; MORI, M.; TAKIGAWA, S.; MATSUURA-ENDO, C; KIN, S. J.; HASHIMOTO, N.; YAMAUCHI, H. Starch phosphorus content in potato (Solanum tuberosum L.) cultivars and its effect on other starch properties. Carbohydrate Polymers, v.68, n.4, p.793-796, 2007.

32 PASCHALL, E. F. Phosphation with organic phosphate salts. In: WHIISTLER, R. E. L. Methods in carbohydrate chemistry. New York: Academic Press, 1964. p.294-296.

33 PASSAUER, L.; LIEBNER, F.; FISCHER, K. Synthesis and properties of novel hydrogels from cross-linked starch phosphates. Macromolecular Symposia, v.244, n.1, p.180-193, 2006.

34 PEREIRA, L. D.; ASCHERI, D. P. R. Efeitos do tempo de reação em função da concentração de tripolifosfato de sódio 
sobre as propriedades físicas e químicas do amido da fruta-de-lobo (Solanum lycocarpum). Revista Agrotecnologia, v. 2, n. 2, p. 82-97, 2011.

35 PRIELER, S.; FISCHER, G. Agricultural by-products associated with biofuel production chains. Netherlands: Elobio, 2009. Report D5.1, 14p. Disponível em: <http://www.elobio.eu/fileadmin/elobio/user/ docs/DeliverableE_5.1.pdf>. Acesso em: 29/06/2014.

36 RINDLAV, A.; HULLEMAN, S. H. D; GATENHOLM, P. Formation of starch films with varying crystallinity. Carbohydrate Polymers. v.34, n.1-2, p.25-30. 1997.

37 SANDHU, K. S., LIM, S. T. Structural characteristics and in vitro digestibility of Mango Kernel Starches (Mangifera indica L.). Food Chemistry, v.107, n.1, p.92-97, 2008.

38 SANDLER, S. R.; KARO, W.; BONESTEEL, J.; PEARCE, E. M. Polymer Synthesis and Characterization. London: Academic Press, 1998.

39 SCHENKEL, E. P.; GOSMANN, G.; ATHAYDE, M. L. Saponinas. In: SIMÕES, C. M.; SCHENKEL, E. P.; GOSMANN, G.; MELLO, J. C. P.; MENTZ, L. A.; PETROVICK, P. R. Farmacognosia: da planta ao medicamento. 3 ed., Porto Alegre: UFGRS/UFSC, 2001. p.597-619.

40 SILVERSTEIN, R. M.; WEBSTER, F.X. Identificação espectrométrica de compostos orgânicos. 6. ed. Rio de Janeiro: Livros Técnicos e Científicos, 2000. 460 p.

41 SINGH, N.; SINGH, J.; KAUR, L.; SODHI, N. S.; GILL, B. S. Morphological, thermal and rheological properties of starches from different botanical sources. Food Chemistry, v.81, n.2, p.219-231, 2003.

42 SITOHY, M. Z.; LABIB, S. M.; EL-SAADANY. S. S.; RAMADAN, M. F.; Physicochemical properties of different types of starch phosphate monoesters. Starch/Stärke. v.52, n.4, p.101-105, 2000.

43 SRICHUWONG, S.; SUNARTI, T. C.; MISHIMA, T.; ISONO, N.; HISAMATSU, M. Starches from different botanical sources II: Contribution of starch structure to swelling and pasting properties. Carbohydrate Polymers, v.62, n.1, p.25-34, 2005.

44 STUART, B. H. Infrared Spectroscopy: Fundamentals and Applications. Inglaterra: John Wiley \& Sons, 2004. 224 p.

45 VIGNEAU, E.; LOISEL, C.; DEVAUX, M. F.; CANTONI, P. Number of particles for the determination of size distribution from microscopic images. Powder Technology, v.107, n.3, p.243-250, 2000.

46 XIAO, H.; LIN, Q.; LIU, G. W.; WU, Y.; TIAN, W.; WU, W.; FU, X. Physicochemical properties of chemically modified starches from different botanical origin. Scientific Research and Essays, v.6, n.21, p.4517-4525, 2011.

47 YASUMURA, P. K.; D'ALMEIDA, L.; PARK, S.W. Multivariate statistical evaluation of physical properties of pulps refined in a PFI mill. O Papel, v.73, n.3, p.59-65, 2012.

48 YUAN, Y.; ZANG, L.; DAI, Y.; YU, J. Physicochemical properties of starch obtained from Dioscorea nipponica Makino comparison with others tubers starch. Journal of Food Engineering, v.82, n.4, p.436-442, 2007.

49 WANG, P.X.; WU, X.L.; DONG-HUA, X.; KUN, X.; YING, T.; XI-BING, D.; WEN-BO, L. Preparation and characterization of cationic corn starch with a high degree of substitution in dioxane-THF-water media. Carbohydrate Research, v. 344, n. 7, p. 851-855, Feb., 2009.

\section{AGRADECIMENTOS}

Agradecemos pelo suporte financeiro à Fundação de Amparo à Pesquisa do Estado de Goiás (FAPEG), à Coordenação de Aperfeiçoamento de Pessoal de Nível Superior (Capes) e ao Conselho Nacional de Desenvolvimento Científico e Tecnológico (CNPq). 